\title{
El enfoque de género en la docencia universitaria desde las voces del alumnado
}

\section{The gender approach in University teaching from the student's standpoint}

\section{ISSN 2071-8748 \\ E-ISSN 2218-3345 \\ (c) (1) (2) \\ BY NC SA}

María A. Martínez-Ruiz ma.martinez@ua.es ORCID: 0000-0001-6028-1877

María E. Urrea-Solano mayra.urrea@ua.es ORCID: 0000-0001-8650-7854

María J. Hernández-Amorós josefa.hernandez@ua.es ORCID: 0000-0002-0931-7114 Universidad de Alicante, España

URI: http://hdl.handle.net/11298/883

DOI: http://dx.doi.org/10.5377/entorno.v0i66.6729

Recibido: 02/08/18 Aprobado: 12/12/18

\section{Resumen}

La incorporación de la perspectiva de género en los procesos de enseñanza y aprendizaje al más alto nivel se perfila, hoy en día, como una de las estrategias más adecuadas para deconstruir las prácticas discriminatorias que enfrentan las mujeres en su cotidianeidad. Por ello, este estudio se planteó con el propósito de conocer la opinión del alumnado sobre el modo en que se aborda el enfoque de género en las aulas universitarias. Para el logro de este objetivo, se contó con la participación de 125 estudiantes de cuatro titulaciones de la Universidad de Alicante (UA), quienes cumplimentaron un cuestionario elaborado ad hoc. La investigación se desarrolló desde un prisma naturalista, realizando un análisis de contenido convencional y sumativo, con la ayuda del software AQUAD 7. De acuerdo con los resultados, aunque la mayoría se mostraba claramente partidaria de abordar este tipo

\section{Abstract}

The incorporation of the gender perspective in the processes of teaching and learning at the highest level is nowadays seen as one of the most appropriate strategies to deconstruct the discriminatory practices that women face in their daily life. Therefore, this study was raised with the purpose of knowing the opinion of students about the way in which the gender approach is addressed in the University classrooms. To achieve this objective, 125 students from four different degrees from the Universidad de Alicante (University of Alicante) who completed an ad hoc prepared questionnaire. The research was developed from a naturalistic prism, performing an analysis of conventional and summative content, assisted by the software AQUAD 7. According to the results and although the majority was clearly in favor of addressing these type of issues in class, their inclusion into the curricula was 
de cuestiones en clase, su incorporación a los planes de estudio era débil y deficitaria. Entre las dificultades aducidas para ello destacaron, especialmente, la rigidez del temario, el carácter polémico del propio contenido o la falta de formación del profesorado. En consecuencia, el alumnado sugirió trabajar este enfoque mediante actividades que fomenten el encuentro, el diálogo y la construcción colectiva en torno a una temática de máxima relevancia por su impacto y trascendencia social. En base a ello, se concluye destacando la necesidad de transversalizar la perspectiva de género en todas las asignaturas y programas del campus, lo que requiere necesariamente de la capacitación y sensibilización previa del profesorado.

\section{Palabras clave}

Enseñanza superior; Estereotipo (psicología); Sociología de la educación; Educación superior. weak and deficient. Among the difficulties alleged for this, they highlighted, particularly, non-flexible agenda, the controversial nature of the content itself, or the lack of academic formation for professors. As a result, students suggested working on this approach through activities that motivates the joint effort, the dialogue and collective construction around a topic of maximum relevance do to its impact and social significance. On this basis it may be concluded and highlight the need of gender perspective transversalization in all courses and academic programs, which necessarily requires the awareness and academic formation of all the professors.

\section{Keywords}

Higher education, Stereotype (psychology), Sociology of education, Higher education.

\section{Introducción}

Los avances sociales y legislativos acaecidos en las últimas décadas están permitiendo visibilizar, quizás como nunca antes, las prácticas sexistas y la desigualdad por razón de género que tienen lugar en nuestra sociedad, en general, y en la academia, de manera particular. Así, es posible comprobar que, a pesar de las metas alcanzadas en el último siglo, la mujer ha de seguir enfrentando múltiples obstáculos en todos los ámbitos de la profesión académica (Wotipka, Nakagawa y Svec, 2018). En este contexto asimétrico, la docencia no constituye una excepción y son diversas las prácticas discriminatorias que siguen sosteniendo la desigualdad en los procesos de enseñanza y aprendizaje que tienen lugar en las aulas universitarias (Mingo y Moreno, 2017). Al sexismo lingüístico, los comentarios despectivos y al discurso paternalista, se han de añadir también los sesgos sexistas que influyen en la evaluación de los aprendizajes, especialmente en aquellos campos de conocimiento tradicionalmente ocupados por los hombres (Xie, Fang y Shauman, 2015). El carácter intangible, sutil y cotidiano de estos mecanismos de exclusión contribuye a la naturalización de las desigualdades y al discurso de la negación por parte de las estudiantes.
Desde este prisma, la incorporación de la perspectiva de género en la agenda universitaria se perfila como uno de los posicionamientos más adecuados para acabar con esta ceguera de género y contribuir al desarrollo de una sociedad más justa y equilibrada (European Institute for Gender Equality, 2016a). En este sentido, la literatura científica y los principales organismos internacionales convienen en señalar que, aplicado a la educación superior, el enfoque de género puede ser entendido como el proceso de análisis crítico del sistema patriarcal, cuyo objetivo es lograr la erradicación de las desigualdades entre mujeres y hombres mediante la promoción de la igualdad en todas las actividades institucionales (European Institute for Gender Equality, 2016b; Lätti, 2017; Morley, 2010; Unesco, 2010). En el caso de la docencia, mediante esta estrategia se pretende incorporar al plan de estudios las experiencias, las necesidades, los intereses y las aportaciones femeninas al conocimiento $y$, de esta manera, fomentar la plena participación de las mujeres en el proceso de aprendizaje.

Pero a pesar de su reconocimiento legal y de los beneficios que comporta para el alumnado, tales como el desarrollo del pensamiento crítico, la interiorización de una nueva conciencia de la realidad y la adquisición de nuevos marcos 
de interpretación (Grünberg, 2011; Lyle-Gonga, 2013), la implementación de la perspectiva de género en las aulas universitarias es notablemente débil y deficitaria (Atchison, 2013; Cassese, Bos y Duncan, 2012). De hecho, en muchos casos, su aplicación depende de la voluntad y del compromiso del profesorado con la igualdad y la justicia social (Serra et al., 2016). Algunas de las razones que parecen explicar esta situación se concentran, especialmente, en la distancia que separa el concepto de la práctica real, ya que la falta de formación, de recursos y de referentes es aducida por la mayor parte del profesorado cuando señala los obstáculos para su aplicación (Hsingchen, 2016). A ello se han de unir, además, las creencias derivadas de la equiparación entre la paridad y la igualdad (Baily y Holmarsdottir, 2015), que llevan a los artífices de su implementación a negar la necesidad de modificar sus prácticas y papeles, a trivializar la igualdad de género e, incluso, a cuestionar la asunción de esta tarea como una responsabilidad profesional (Lombardo y Mergaert, 2013; Verge, Ferrer-Fons y González, 2018).

No obstante, en aquellos contextos donde su aplicación ha sido exitosa, el impacto generado en el alumnado ha sido amplio y notable. Así se evidenció, por ejemplo, en la investigación de Yu (2018), en la que el estudio y debate colectivo sobre literatura y género fue especialmente efectivo para incrementar el nivel de conciencia de género de los alumnos y las alumnas. Otra experiencia, en este caso con estudiantes de Sociología, vino a señalar que la incorporación del conocimiento feminista al plan de estudios les permitió obtener una identidad profesional más profunda y completa (Abbas, Ashwin y McLean, 2016). En el caso de la formación inicial del profesorado, también se ha comprobado que su implementación, además de generar una profunda satisfacción entre el alumnado, resulta especialmente efectiva para el cuestionamiento de las normas sociales, la mejora del futuro desempeño profesional y su empoderamiento como diásporas de la ideología de género (Camacho-Miñano y Girela-Rejón, 2017; Donoso-Vázquez y Velasco-Martínez, 2013). Por ello, el objetivo de la presente investigación fue conocer la visión del alumnado sobre el tratamiento que las cuestiones de género reciben en las aulas universitarias. A tal efecto, se plantearon las siguientes cuestiones de investigación: (1) ¿Cómo se aborda, desde la perspectiva del alumnado, la igualdad de oportunidades de género en la docencia universitaria de las distintas titulaciones de la UA? (2) ¿Qué opina el alumnado de la UA sobre la importancia que adquiere el tratamiento de este tema en las aulas universitarias? (3) ¿Qué dificultades aprecia para su implementación? (4) ¿Qué propuestas sugiere para mejorar el tratamiento de la igualdad de género en su titulación?

\section{Metodología}

El estudio se desarrolló desde un prisma naturalista de investigación, por cuanto el interés radicaba en interpretar las voces de los participantes en su contexto natural (Cho y Lee, 2014).

\section{Participantes}

Se contó con la participación de 125 estudiantes, siendo el $51,2 \%$ mujeres. La mayoría $(91,2 \%$ ) tenía una edad comprendida entre los 18 y 23 años y estaba cursando Ciencias de la Actividad Física y el Deporte (45,6\%). Por su parte, el 27,2 \% pertenecía al Grado de Criminología, el $22,4 \%$ era alumnado del Grado de Maestro en Educación Primaria y, por último, un 4,8 \% estaba matriculado en Gestión y Administración Pública. Más de la mitad de la muestra $(58,4 \%)$ se encontraba en el segundo curso de sus estudios.

\section{Instrumento y proceso de recogida de datos}

Para la recogida de datos se diseñó un cuestionario compuesto por cinco cuestiones de carácter abierto (Anexo 1), que fue validado por tres expertas del área de Didáctica y Organización Escolar. Dado que este trabajo forma parte de otro de mayor magnitud, dirigido a comparar las opiniones del profesorado y alumnado de la UA sobre la incorporación de la perspectiva de género a la docencia universitaria, se aprovechó la entrevista con el personal docente para plantear la posibilidad de encuestar a sus estudiantes. Conseguida su autorización preceptiva, se procedió a recopilar los datos del alumnado, quien fue informado, en todo momento, de las pretensiones del estudio, del carácter voluntario de su participación, así como del anonimato y confidencialidad de sus respuestas. La cumplimentación del instrumento llevó un tiempo medio aproximado de 20 minutos.

\section{Procedimiento de análisis de datos}

Una vez recogidos los cuestionarios, se procedió a su transcripción para posteriormente codificarlos. El procedimiento de transcripción permitió, de entrada, clasificar 
y organizar la información de manera mental, lo que favoreció el planteamiento posterior del marco de códigos y categorías. Tanto es así que este proceso, sumado a la lectura reiterativa de las aportaciones de los encuestados, fue decisivo en la configuración de dicho instrumento de interpretación.

Se realizó un análisis de contenido convencional (Hesieh y Shannon, 2005), en un proceso inductivo, en el que la información emergió directamente de las voces de los participantes. Este análisis inferencial se complementó con un análisis de frecuencias, tendente no a inferir significados sino a contabilizarlos. Ciertamente, el análisis de contenido - como método de investigación cualitativa - se centra en valorar el lenguaje utilizado como vía de comunicación, conscientes de que adquiere un significado determinado en un contexto particular.

El proceso de codificación se realizó con el apoyo del software AQUAD 7 (Huber y Gürtler, 2013), que facilitó significativamente las tareas de organización, síntesis y categorización de la información en unidades de significado de amplitud variable (categorías y códigos). En realidad, la lógica interna de este programa permite que los investigadores e investigadoras realicen un análisis riguroso de los datos para facilitar la interpretación, siguiendo un proceso recursivo que conecta la propuesta de categorías y códigos con las cuestiones de investigación.

El mapa de categorías y códigos fue validado por tres expertas en investigación educativa. La primera propuesta fue modificada ligeramente durante el proceso de codificación, ya que la información emergente favoreció la consideración de nuevos códigos. La propuesta definitiva queda recogida en el Anexo 2. En ella, se aprecia la clasificación de la información en torno a cuatro categorías: (1) Tratamiento temática, (2) Opinión relevancia, (3) Dificultades y (4) Propuestas. El establecimiento de los códigos para cada una de ellas nos permitió ahondar en su significado.

\section{Resultados}

Los resultados quedan organizados en función de las cuatro categorías establecidas. En su exposición, se presentan los datos de carácter inferencial —selección de algunas de las narrativas más significativas de cada categoríay descriptivo. No obstante, en la figura 1 se muestra una panorámica general del conjunto de datos del estudio. En ella, se atiende a la frecuencia absoluta (FA) de cada código, entendida como el número de veces que el alumnado hace referencia a dicha unidad de información.

Figura 1. Frecuencia absoluta de los códigos de la investigación

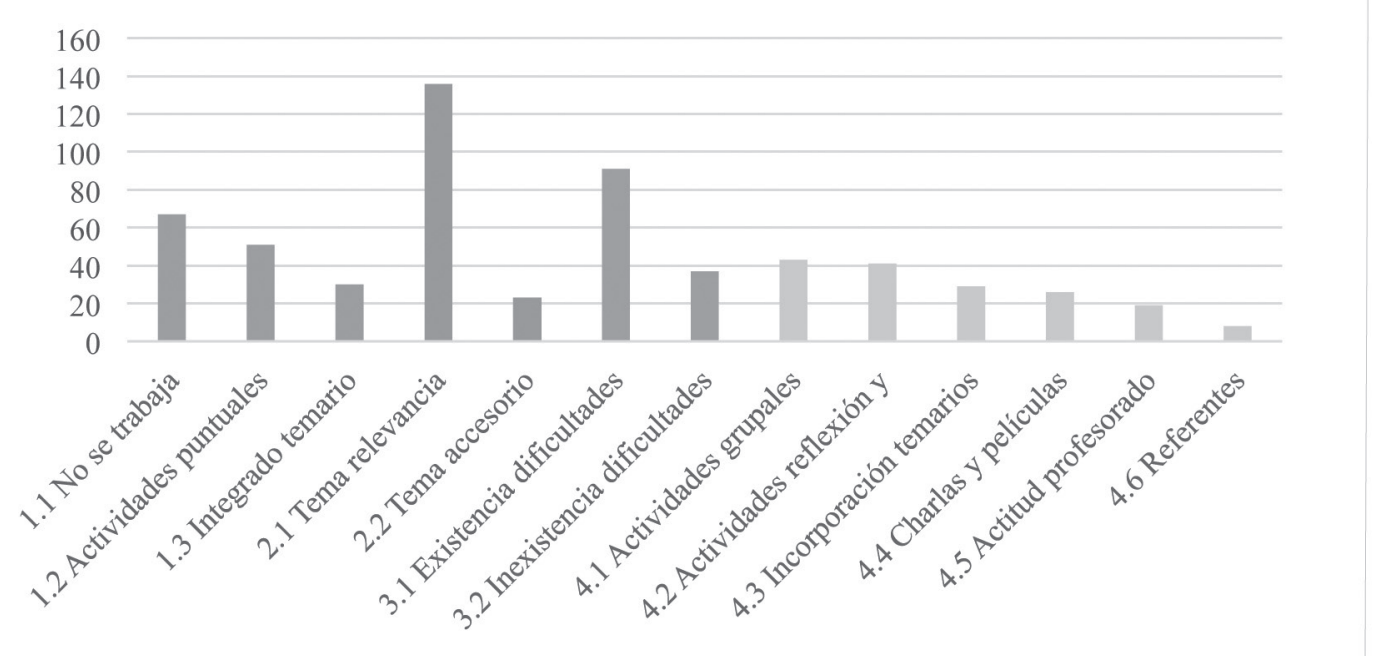


Los niveles más altos de frecuencia, considerados en la figura 1, permiten afirmar que los participantes incidieron repetidamente sobre la idea de que la igualdad de oportunidades de género es un tema de considerable relevancia, sugerente deabordaje en la educación superior. Sin embargo, en la mayoría de los casos, confirmaron que o bien no se trabajaba o se hacía de manera puntual. Reconocieron, asimismo, las dificultades que entraña integrarlo en los programas de docencia universitaria y establecieron una serie de propuestas relacionadas eminentemente con la creación de grupos mixtos, así como con el diseño y desarrollo de actividades de carácter reflexivo.

Cat. 1. Tratamiento temática

Cat. 2. Opinión relevancia Cat. 3. Dificultades

Cat. 4. Propuestas

\section{Categoría 1. Tratamiento temática}

Esta categoría es reveladora del modo en que se había trabajado la temática objeto de estudio en las aulas de los participantes. Su análisis permite comprobar la existencia de tres matices claramente diferenciados, de los que se deriva la propuesta de códigos, cuyos datos descriptivos quedan recogidos en la tabla 1.

Tabla 1. Códigos inferenciales de la Categoría 1: dimensiones cuantitativas

\begin{tabular}{lcc}
\hline \multicolumn{1}{c}{ Códigos } & FA & FA (\%) \\
\hline 1.1 No se trabaja & 67 & 45,3 \\
1.2 Actividades puntuales & 51 & 34,5 \\
1.3 Integrado temario & 30 & 20,3 \\
\hline
\end{tabular}

El código sobre el que más se incidió evidencia la ausencia de atención y trabajo específico del tema de estudio. Las narrativas seleccionadas son muestra de ello:

Nunca he tenido asignaturas donde se haya abordado la igualdad de oportunidades de género. (Alu_064)

No hemos abordado el tema de manera específica en ninguna asignatura. (Alu_120)

En los casos en los que se había abordado, parece predominar su tratamiento puntual por medio de actividades como debates o vídeos planteados al margen de los diseños propiamente curriculares:

Hemos abordado la igualdad de género en debates que se han creado en clase, pero sin darle mayor importancia. Además, hemos realizado un trabajo de discriminación a la mujer, dándose el caso de la escasez de oportunidades de género, sobre todo en el ámbito laboral. (Alu_004)
Únicamente, en la asignatura de Teoría de la Actividad Física y del Deporte, en primero, se leyeron varios artículos que hablaban sobre el tema, pero no se profundizó en ellos. (Alu_070)

Nos han puesto vídeos donde salen mujeres explicando el tema, pero no lo hemos abordado. (Alu_106)

En último término, se reconocieron algunas voces que afirman que la temática había sido incluida en las clases de manera específica y como contenido propio de los temarios:

En una asignatura de primero, Cambios Sociales. En ella sí había un tema exclusivo donde se abordaba la igualdad de género [...]. (Alu_045)

Hemos tratado el tema del deporte y la mujer, en el que hemos visto las diferencias existentes en el deporte profesional entre hombres y mujeres. En concreto, aspectos como sueldo, expectación, seguimiento en los medios de comunicación, etc. (Alu_115) 
Categoría 2. Opinión relevancia

En esta categoría, se incluyen las opiniones del alumnado en torno a la importancia que concede al tratamiento de la igualdad de oportunidades de género en las aulas universitarias. Estas evidencian la existencia de dos posturas claramente diferenciadas, siendo la mayoritaria la que reconoce un valor real al tema por diversos motivos que quedan recogidos, a modo de subcódigos, en la tabla 2.

Tabla 2. Códigos inferenciales de la Categoría 2: dimensiones cuantitativas

\begin{tabular}{lcc}
\hline \multicolumn{1}{c}{ Códigos } & FA & FA (\%) \\
\hline 2.1 Tema relevancia & 136 & 85,5 \\
2.1.1 Formación integral & 38 & 23,9 \\
2.1.2 Impulso cambio & 33 & 20,8 \\
2.1.3 Lucha discriminación & 29 & 18,2 \\
2.1.4 Mejora capacitación & 21 & 13,2 \\
2.1.5 Tema actualidad & 15 & 9,4 \\
2.2 Tema accesorio & 23 & 14,47 \\
\hline
\end{tabular}

Entre los argumentos argüidos por los estudiantes en defensa del trabajo de la igualdad de oportunidades de género en la educación superior, aquel sobre el que incidieron de forma más reiterativa fue el impacto que posee en su formación. Manifestaron que tener preseente este y de otros aspectos de carácter social confiere a los estudios universitarios un cariz más holístico y no tan academicista:

Sí, creo que es importante porque debemos ser conscientes de la realidad para poder ser críticos y reflexionar. Para ello, debemos conocer el enfoque de género. (Alu_048)

Creo que sí es importante incluir dicho enfoque, ya que al final es aquí, en la universidad, donde se "culmina" la formación de las personas y no solo en el ámbito académico, también en el ámbito de la personalidad y los valores. (Alu_082)

En relación con su formación, y aunque en menor medida, destacaron que el tratamiento de esta temática contribuye a mejorar su cualificación profesional. Son los futuros docentes los que, especialmente, se detuvieron a hacer esta valoración por cuanto reconocen la trascendencia social que tiene su labor:

Desde mi punto de vista, como futuros educadores, debemos inculcar a los alumnos la igualdad de género, ya que tanto hombres como mujeres debemos tener las mismas posibilidades de avanzar académicamente y de encontrar en un futuro un buen puesto de trabajo en nuestra disciplina, independientemente del género. (Alu_124)

Ahora bien, buena parte del alumnado destacó que es un contenido que debe trabajarse no únicamente en la educación superior, sino desde los primeros estadios de la educación básica:

Creo que es importante no solo en la universidad, sino también en educación primaria y educación secundaria, ya que considero que es una vía para ir disminuyendo la desigualdad entre hombres $y$ mujeres. (Alu_021)

Defendieron, de igual modo, que este tipo de formación se convierte en propulsora del cambio social que se requiere:

Sí, como en todos los ámbitos, porque es importante para que la sociedad avance y mejore. (Alu_064)

Sí, porque hay gente (alumnos y profesores) que no le da importancia, todavía viven con un pensamiento machista y retrógrado y, con la información adecuada, esto puede ayudar a cambiar y avanzar. (Alu_008) 
Por ejemplo, alegaron que se convertiría en un revulsivo para el arraigo de creencias, valores y actitudes coherentes con las estructuras plurales, equitativas y diversas que demanda la sociedad actual, capaz de erradicar situaciones de discriminación por razón de género:

Creo que es un tema que se debería tratar muchísimo más, ya que no por ser mujeres debemos aguantar acosos, la brecha salarial y un millón más de discriminaciones y machismos que sufrimos todas a diario. (Alu_003)

Sí, porque así muchos podrían cambiar su forma de tratar a las mujeres y no habría tanta discriminación en el futuro. (Alu_016)

Finalmente, el aspecto sobre el que menos incidieron fue sobre la vigencia del tema. Sin embargo, en algunas de sus respuestas, se aduce que la universidad como institución debe formar para el presente $y$, especialmente, para el futuro, lo que exige considerar este y otros temas de candente actualidad:

Creo que es muy importante tratar esto, ya que es un tema que está muy presente en la sociedad. (Alu_005)

Se debe trabajar, ya que actualmente es un tema que afecta a toda la sociedad, focalizando el problema en las desigualdades sufridas entre los dos géneros. Por ello, qué mejor sitio que en la universidad. (Alu_028)
Las aportaciones presentadas hasta el momento son prueba de la actitud mayoritaria de defensa y compromiso con la incorporación de la igualdad de oportunidades de género en la docencia universitaria. Sin embargo, también se ha apreciado la existencia de opiniones distintas. Algunas de ellas son radicalmente contrarias a las anteriores, al manifestar que no es una temática que deba trabajarse en la universidad porque no existe desigualdad por razón de género. También es cierto que, en este conjunto, se aprecia la existencia de voces que apoyan el trabajo de la igualdad únicamente cuando esta pueda verse afectada:

No lo abordaría, ya que es un tema que ya debería estar inculcado en la sociedad como algo normal. (Alu_007)

Se tiene que abordar siempre y cuando sea necesario. (Alu_073)

Tampoco es necesario que se trabaje, ya que en mi opinión ya existe una igualdad de oportunidades, ya que da igual que seas chico o chica. Lo importante es que seas alumno. (Alu_095)

\section{Categoría 3. Dificultades}

Conocida la valoración de los estudiantes respecto a la inclusión de la perspectiva de género en los currículos, se presentan los resultados relativos a las dificultades que consideran entraña dicha incorporación. Como se muestra en la tabla 3, repararon más en la existencia de obstáculos que en la consideración de la ausencia de límites.

Tabla 3. Códigos inferenciales de la categoría 3: dimensiones cuantitativas

\begin{tabular}{lcc}
\hline \multicolumn{1}{c}{ Códigos } & FA & FA (\%) \\
\hline 3.1 Existencia dificultades & 91 & 71,09 \\
3.1.1 Rigidez temarios & 34 & 26,6 \\
3.1.2 Tema controvertido & 26 & 20,3 \\
3.1.3 Falta formación y & 19 & 14,8 \\
sensibilización profesorado & & \\
3.1.4 Escasez mujeres & 12 & 9,4 \\
3.2 Inexistencia dificultades & 37 & 28,9 \\
\hline
\end{tabular}


La dificultad sobre la que insistieron con mayor énfasis fue en la rigidez de los temarios, lo que supone escaso tiempo para trabajar este contenido:

Que no es un tema muy vinculado con muchas asignaturas o siguen la programación y no se paran a tratar estos temas. (Alu_024)

Muchas veces lo vemos como un tema menos importante o que no está dentro de la programación, por eso el tiempo puede ser un impedimento. (Alu_059)

Como apenas hay tiempo para abordar todos los contenidos de las asignaturas, no se aborda el tema de la igualdad de forma específica. (Alu_120)

De igual modo, reconocieron que otra de las limitaciones para su implementación es la controversia que puede llegar a generar en clase, dada la existencia de opiniones muy variadas al respecto:

Al ser muchos alumnos, existen muchos puntos de vista, por lo que podrían surgir conflictos entre alumnos, incluso entre un alumno y el/la profesor/a. (Alu_013)

Es un tema peliagudo, para el que se tiene una gran disparidad de opiniones, con las que siempre hay conflicto. (Alu_028)

Puede ser un tema malinterpretado o desafortunado en el que alguna persona pueda sentirse ofendida o menospreciada por una comunicación confusa o incorrecta. (Alu_111)

A su vez, apuntaron que la falta de formación y de sensibilización del profesorado con este tema se constituye como otro impedimento importante a tener en cuenta:
La falta de recursos por parte del profesorado, que no sabe cómo abordarlo si no es dando el tema como tal. (Alu_044)

Que el profesorado se centra en impartir su materia y ya está. Se piensa que su asignatura no tiene nada que ver con esto. (Alu_037)

Yo creo que la mayor dificultad es la ideología de algunos profesores, que no creen en la igualdad de género. (Alu_115)

En último término, opinaron que la escasez de mujeres puede justificar también el hecho de que este tema se trabaje escasamente en las aulas de educación superior:

Creo que el mayor problema se encuentra en la gran diferencia que existe entre chicos y chicas en cuanto a número, ya que por ejemplo actualmente en clase hay ocho chicas de 42 alumnos. (Alu_090)

En nuestra carrera es difícil tratar la igualdad porque de 100 alumnos, solo 20 son mujeres, y la gran parte de profesores son hombres. (Alu_114)

La principal dificultad que encontramos en esta carrera es que, aunque trabajamos la igualdad de género, somos muy poquitas mujeres y no llegamos a ningún lado. (Alu_122)

\section{Categoría 4. Propuestas}

En última instancia, la cuarta de las categorías integra aquellas aportaciones de los participantes que engloban una serie de sugerencias para incluir el enfoque de género en la docencia universitaria.

Tabla 4. Códigos inferenciales de la categoría 4: dimensiones cuantitativas

\begin{tabular}{lcc}
\hline \multicolumn{1}{c}{ Códigos } & FA & FA (\%) \\
\hline 4.1 Actividades grupales & 43 & 25,9 \\
4.2 Reflexión y sensibilización & 41 & 24,7 \\
4.3 Incorporación temarios & 29 & 17,5 \\
4.4 Charlas y películas & 26 & 15,7 \\
4.5 Actitud profesorado & 19 & 11,4 \\
4.6 Referentes & 8 & 4,8 \\
\hline
\end{tabular}


Como muestra la tabla 4, la propuesta más apoyada fue la creación de grupos de trabajo mixtos para la realización de las distintas actividades propuestas:

Plantear ejercicios donde cooperen hombres y mujeres, sin considerar a la mujer inferior en cuanto a sus capacidades. (Alu_049)

Tomas de concienciación o trabajos comunes con otras ramas de la educación, con el fin de cooperar e igualar el número de miembros de ambos sexos, para poder después utilizar feedback y apreciar las discrepancias que puedan surgir. (Alu_107)

Siempre fomentaría la participación mixta en los grupos e intentaría que hiciesen el mismo papel a la hora de realizar actividades. (Alu_090)

Otro planteamiento recurrente fue el diseño y desarrollo de actividades que generen actitudes reflexivas y que promuevan la sensibilización con el tema:

Hacer debates y reflexionar. Poner ejemplos claros de dónde se da este asunto. (Alu_037)

Haría debates sobre estos temas y actividades de reflexión en las que plasmaría que todos somos aptos para todo. (Alu_047)

Hacer una campaña de sensibilización, como la que preparamos para Psicología, y llegar a un debate que nos proporcione una conclusión. (Alu_061)

Plantearon, asimismo, la conveniencia de incluir la igualdad de oportunidades de género como contenido específico en los temarios de todas o de alguna de las asignaturas:

Que el temario dado en clase tuviera una perspectiva de género. Es decir, al hablar de la historia, que se viera tanto el papel de la mujer como el del hombre. (Alu_012)

En cada asignatura, tener un bloque donde se trabajara este tipo de contenidos. (Alu_027)

Que algunas asignaturas incluyan una parte específica donde se hable de las diferencias de género, con el fin de concienciar y divulgar. (Alu_110)
Sugirieron también la realización de actividades en gran grupo y en las que pueda participar todo el alumnado de la UA como, por ejemplo, charlas y visionado de películas:

Charlas de concienciación con debates incluidos, animándonos a los alumnos y alumnas a participar en ellos $y$, sobre todo, concienciando de que estas cosas siguen pasando hoy en día, y que no es algo lejano, ni en el tiempo ni en el lugar. (Alu_003)

Proyectaría vídeos en los que se viera reflejada esta desigualdad que actualmente se mantiene para hacernos reflexionar. (Alu_042)

En algunos casos, propusieron un cambio de actitud en los docentes, quienes consideran que deberían estar más sensibilizados con el tema y otorgar más tiempo al trabajo este. Destacaron, de manera convencida, que su ejemplo es una de las principales vías de aprendizaje:

Los docentes deberían modificar su lenguaje, su forma de expresión y de tratar a las mujeres. Creo que a veces nos sentimos en algunos momentos discriminadas por ellos. (Alu_122)

Darle tiempo a estos temas en las clases y relacionarlos con temas actuales. (Alu_005)

Pienso que lo más importante es transmitirlo en el día a día, mediante tus comentarios, de los ejemplos o ilustraciones que utilizan para dar las clases, de su manera de actuar o de comportarse con los alumnos. (Alu_056)

En último término, entendían oportuno dar a conocer testimonios de mujeres sobre sus trayectorias para inspirar su desempeño profesional futuro:

En el caso de CAFD, trataría de resaltar la importancia de la mujer en el deporte. Es fundamental reconocer el trabajo de muchas deportistas de élite que, por su condición, no obtienen el mismo reconocimiento que sus compañeros. Esto implantaría la idea en mucha gente de que la igualdad es una realidad. (Alu_072)

En esta línea, también consideraron importante conocer testimonios de mujeres que han sufrido la discriminación, en algún ámbito, por razón de género: 
Intentaría traer a gente que haya padecido una desigualdad de género a nivel estudiantil y en el ámbito deportivo para abrir mentes y conciencias. (Alu_106)

\section{Discusión}

Reconociendo el valor del tratamiento de las cuestiones de género en las aulas universitarias para el logro de una sociedad más justa y equitativa, el presente estudio se desarrolló con el objetivo de conocer la opinión del alumnado acerca de la incorporación de este enfoque en las titulaciones cursadas. A la vista de los resultados anteriormente analizados, todo parece indicar que este tipo de contenidos sigue sin tener el reconocimiento y el espacio que merecen en el plan de estudios, limitando su presencia a un mero abordaje anecdótico y superficial. Esta práctica coincide con la tendencia predominante en las instituciones de educación superior, donde la formación transversal en género es notablemente escasa y deficitaria (Atchison, 2013; Serra et al., 2016). Parece por tanto que, a pesar de las recomendaciones institucionales y de las ventajas que presenta para el desarrollo personal y la capacitación profesional del alumnado (Grünberg, 2011; Lyle-Gonga, 2013), el conocimiento sobre el género sigue estando ausente en los procesos de enseñanza y aprendizaje al más alto nivel.

Pero aunque su integración en los planes de estudio sea mínima, el alumnado se muestra claramente partidario de su tratamiento en las aulas, otorgándole una gran relevancia por tratarse de un aspecto clave para la formación personal y cívica de todo ser humano y por representar un revulsivo para la mejora profesional y para el avance social. Esta valoración positiva es también reconocida en otras investigaciones, donde el alumnado es capaz de reconocer los beneficios que este tipo de contenidos aporta para su crecimiento personal y profesional (Abbas et al., 2016; Donoso-Vázquez y Velasco-Martínez, 2013; Yu, 2018). Así, aunque en un principio no se sienta atraído por estas cuestiones, el trabajo y la inmersión en el aula le permiten comprender que este conocimiento resulta especialmente relevante para su futuro desempeño profesional y para su formación axiológica, llegando incluso a considerarla como una materia que debería ser obligatoria (Camacho-Miñano y Girela-Rejón, 2017).

A pesar de ello, es interesante subrayar la existencia de quienes consideran que este tipo de contenidos no han de ser abordados en la formación universitaria, ya que entienden que la igualdad entre mujeres y hombres es ya una realidad consumada. Algunas de las razones que pueden explicar este posicionamiento se derivan de la confusión terminológica entre paridad e igualdad, equívoco que, como señalan Baily y Holmarsdottir (2015), puede acabar distorsionando la meta que se ha de alcanzar por cuanto lo cuantitativo ofrece una visión sesgada de las desigualdades que sostienen el sistema patriarcal. Otro de los motivos que explica esta resistencia se centra en el carácter controvertido del contenido. De acuerdo con el alumnado, el género constituye un tema susceptible de debate y discusión, por lo que se considera que este ha de permanecer en la esfera privada de cada individuo. Esta división tradicional entre las áreas de conocimiento públicas y privadas también ha sido identificada por verge et al. (2017), para quienes esta diferenciación solo sirve para reforzar las prácticas discriminatorias contra la mujer. A la vista de tales creencias, parece necesaria una formación que permita ir más allá de la paridad y desvelar el verdadero significado e impacto que la igualdad de género tiene, tanto a nivel personal como social.

En cuanto a los elementos que, según el alumnado, entorpecen el abordaje de estas cuestiones en los procesos de enseñanza y aprendizaje universitarios, estos parecen ser múltiples y variados. Así, se refieren a la escasa flexibilidad de un temario - caracterizado por la abundancia de contenidos, la falta de tiempo y la rigidezy a la naturaleza polémica del contenido, aspectos que vienen a dificultar, en muchos casos, el establecimiento de posibles relaciones con las cuestiones de género. Hallazgos similares han sido identificados por Camacho-Miñano y Girela-Rejón (2017) y Verge et al. (2017), quienes señalan, además, que las dificultades que encuentran a la hora de identificar las prácticas sexistas, la actitud adaptativa y conformista que suelen presentar ante este tipo de hechos y el desconocimiento que muestran hacia la ideología de género pueden ser algunas de las razones que les lleven a distanciarse de estas cuestiones. Otra de las barreras que parecen identificar para trabajar este tipo de contenidos en clase es la falta de capacitación y sensibilización del profesorado. Resultados que se alinean con lo indicado por la literatura científica, al considerar que la escasa formación docente en estas cuestiones se convierte en uno de los principales obstáculos para la incorporación de la perspectiva de género a los procesos de enseñanza y aprendizaje universitarios (Hsingchen, 2016). 
Como propuestas para superar estas resistencias, el alumnado propone, especialmente, trabajar este tipo de contenidos mediante actividades que sean capaces de estimular la reflexión, el encuentro y el debate. Dinámicas dialógicas que, tal y como se ha puesto de manifiesto en otros estudios (Hsingchen, 2016; Yu, 2018), resultan especialmente efectivas para despertar e inspirar la conciencia de género y visibilizar lo invisible.

\section{Conclusiones}

Ante este panorama, parece quedar fuera de toda duda la necesidad de implementar medidas eficaces para garantizar el adecuado tratamiento de las cuestiones de género en los planes de estudio universitarios. La consolidación de estos contenidos no se trata de un reconocimiento académico sin más, sino del aprovechamiento de una contribución científica determinante para lograr un mundo más justo y equitativo (Donoso-Vázquez y Velasco-Martínez, 2013). Además, la academia, como espacio de reflexión, conocimiento e innovación, se convierte en un entorno privilegiado para el desarrollo integral de las capacidades de todas las personas, y, por ello, se han de movilizar esfuerzos para hacer efectiva la incorporación de la perspectiva de género de manera transversal en los enfoques y contenidos de todas las asignaturas y programas.

Resulta preocupante, por otra parte, que dadas las fortalezas que presenta la perspectiva de género para la formación del alumnado, así como la predisposición que este muestra hacia este tipo de contenidos, exista tan poco espacio en el plan de estudios para su difusión. En consecuencia, se ratifica que la confianza excesiva en la transversalidad, como única estrategia para abordar las cuestiones de género, está resultando ineficaz, ya que en la práctica se acaba convirtiendo en una coartada para el inmovilismo. Por ello, resulta urgente repensar la transversalidad y diseñar un nuevo modelo, más abierto y flexible, donde sea posible comprehender este tipo de cuestiones. En este ejercicio reflexivo sería conveniente que participaran todos los agentes y sectores de la comunidad universitaria, con el objetivo de lograr el compromiso y la colaboración que requiere la reformulación de las titulaciones, de las materias y de los planes de estudio desde un enfoque de equidad y justicia social.

En última instancia, sería preciso considerar que la transversalización de la igualdad, desde una perspectiva de género interseccional, exige previamente la formación y la sensibilización del personal docente encargado de su aplicación. El objetivo de esta capacitación pasa por dotarle de los instrumentos más adecuados para identificar la discriminación de género en el ejercicio de su docencia y, en última instancia, revertirla. Solo de este modo se podrán ofrecer herramientas de conocimiento crítico para que el alumnado sea capaz de emplearlas en su futuro desempeño profesional y en su vida cotidiana, como miembro integrante y propulsor de una sociedad más justa y democrática.

\section{Agradecimientos}

Este estudio se ha realizado en el marco del proyecto de cooperación universitaria para el desarrollo denominado "14/17 Red de Investigación en diferenciales de Género en las Universidades Iberoamericanas", subvencionado por el Vicerrectorado de Relaciones Internacionales de la Universidad de Alicante y el Ministerio de Sanidad, Servicios Sociales e Igualdad.

\section{Referencias}

Abbas, A.; Ashwin, P., \& McLean, M. (2016). "The influence of curricular content on English sociology students' transformations: the case of feminist knowledge". Teaching in Higher Education, 21(4), 1-15. DOI: 10.1080/13562517.2016.1155551

Atchison, A.L. (2013). "The practical process of gender mainstreaming in the Political Science curriculum". Politics \& Gender, 9(2), 228-235. DOI: 10.1017/ S1743923X13000081

Baily, S., \& Holmarsdottir, H.B. (2015). "The quality of equity? Reframing gender, development and education in the post-2020 landscape". Gender and Education, 27(7), 828-845. DOI: 10.1080/09540253.2015.1103842

Camacho-Miñano, M.J., \& Girela-Rejón, M.J. (2017). “Evaluación de una propuesta formativa sobre género en Educación Física para estudiantes de Ciencias de la Actividad Física y el Deporte". Cultura, Ciencia y Deporte: revista de Ciencias de la Actividad Física y del Deporte de la Universidad Católica de San Antonio, 36, 195-203.

Cassese, E.C.; Bos, A.L., \& Duncan, L.E. (2012). "Integrating gender into the Political Science core curriculum". PS: Political Science and Politics, 45(2), 238-243. DOI: $10.1017 /$ S1049096512000042 
Cho, J.Y., \& Lee, E. (2014). "Reducing confusion about grounded theory and qualitative content analysis: Similarities and differences". The Qualitative Report, 19(32), 1-20.

Donoso-Vázquez, T., \& Velasco-Martínez, A. (2013). “¿Por qué una propuesta de formación en perspectiva de género en el ámbito universitario?". Profesorado. Revista de Currículum y Formación del Profesorado, 17(1), 71-88.

European Institute for Gender Equality. (2016a). Positive impact of gender mainstreaming in academia and research institutions. Luxembourg: Publications Office of the European Union. Recuperado el 12 de junio de 2018 de http://eige.europa.eu/sites/default/files/ documents/mh0116826enn_without_blank_pages.pdf

European Institute for Gender Equality. (2016b). Gender equality in academia and research. Luxembourg: Publications Office of the European Union. Recuperado el 3 de junio de 2018 de http://eige.europa.eu/sites/ default/files/2016.5791_eige_gender_equality_in_ academia.pdf

Grünberg, L. (2011). "From gender studies to gender IN studies and beyond". En L. Grünberg (Ed.), From gender studies to gender IN studies. Case studies on gender-inclusive curriculum in higher education (pp. 7-15). Bucarest: Unesco-Cepes.

Hsieh, H.F., \& Shannon, S. (2005). "Three approaches to qualitative content analysis". Qualitative Health Research, 15(9), 1277-1288. DOI: 10.1177/1049732305276687

Hsingchen, Y. (2016). "Transforming concepts into practices: mainstreaming gender in higher education". Asian Journal of Women's Studies, 22(4), 392-413. DOI: 10.1080/12259276.2016.1242940

Huber, G.L., \& Gürtler, L. (2013). AQUAD 7. Manual: The analysis of qualitative data. Tübingen, Germany: Authors. Recuperado el 14 de marzo de 2018 de http://www. aquad.de/materials/manual_aquad7/manual-e.pdf

Lätti, J. (2017). "Individualized sex equality in transforming Finnish academia". European Educational Research Journal, 16(2-3), 258-276. DOI: $10.1177 / 1474904116668184$
Lombardo, E., \& Mergaert, L. (2013). “Gender mainstreaming and resistance to gender training: a framework for studying implementation". NORA - Nordic Journal of Feminist and Gender Research, 21(4), 296-311. DOI: 10.1080/08038740.2013.

Lyle-Gonga, M. (2013). "A critical analysis of gender mainstreaming". Politics \& Gender, 9(2), 209-213. DOI: 10.1017/S1743923X13000056

Mingo, A., \& Moreno, H. (2017). "Sexismo en la Universidad". Estudios Sociológicos, 35(105), 571-595. DOI: 10.24201/ es.2017v35n105.1434

Morley, L. (2010). "Gender mainstreaming: myths and measurement in higher education in Ghana and Tanzania". Compare, 40(4), 533-550. DOI: 10.1080/03057925.2010.490377

Serra, P.; Soler, S.; Prat, M.; Vizcarra, M.T.; Garay, B., \& Flintoff, A. (2016). "The (in) visibility of gender knowledge in the Physical Activity and Sport Science degree in Spain". Sport, Education and Society, 23(4), 324-338. DOI: 10.1080/13573322.2016.1199016

Unesco (2010). Gender issues in higher education. Bangkok: Unesco. Recuperado el 5 de junio de 2018 de http:// unesdoc.unesco.org/images/0018/001898/189825e.pdf

Verge, T.; Ferrer-Fons, M., \& González, M.J. (2018). “Resistance to mainstreaming gender into the higher education curriculum". European Journal of Women's Studies, 25(1), 86-101. DOI: $10.1177 / 1350506816688237$

Wotipka, C.M.; Nakagawa, M., \& Svec, J. (2018). "Global linkages, the higher education pipeline, and national contexts: the worldwide growth of women faculty, 19702012". International Journal of Comparative Sociology, 59(3), 212-238. DOI: 10.1177/0020715218780475

Xie, Y.; Fang, M., \& Shauman, K. (2015). "STEM Education". Annual Review of Sociology, 41, 331-357. DOI: 10.1146/ annurev-soc-071312-145659

Yu, Y. (2018). "Using 'classic Reading instruction' to raise students' gender awareness: student's perceptions of their learning experiencies at a Taiwanese university". Asian Journal of Women's Studies, 24(1), 88-105. DOI: 10.1080/12259276.2017.1421292 
Anexo 1. Instrumento de recogida de datos

\section{La perspectiva de género en la docencia universitaria}

Este cuestionario forma parte de una investigación dirigida a conocer las experiencias y la opinión del alumnado sobre el tratamiento que el género recibe en las titulaciones de la Universidad de Alicante (UA). Es por ello que, reconociendo la importancia de tu participación, te informamos de que los datos recogidos se tratarán de manera totalmente anónima, invitándote a que des respuesta a cada pregunta de la forma más sincera posible.

\section{Muchas gracias por tu colaboración}

Sexo:

\section{Edad:}

Titulación que se está cursando:

\begin{tabular}{|l|l|l|l|}
\hline $\mathbf{1}$ & $\mathbf{2}$ & $\mathbf{3}$ & $\mathbf{4}$ \\
\hline
\end{tabular}

Curso:

1.Comenta cómo y cuándo se ha abordado la igualdad de oportunidades de género en las asignaturas que has cursado en la UA.

2. Y en esta asignatura en concreto, ¿cómo y cuándo se ha abordado esta cuestión?

3. ¿Crees que es importante incluir el enfoque de género en las clases en la universidad? ¿Por qué?

4.¿Qué dificultades o problemáticas encuentras para trabajar la igualdad de género en las clases de la universidad?

5. ¿Qué propuestas harías para trabajar este tipo de contenidos en las aulas universitarias? 


\section{Anexo 2. Marco de referencia para la interpretación de datos}

\section{Marco de categorías y códigos}

\section{Categoría 1. Tratamiento temática}

Se incluyen las aportaciones del alumnado referidas al grado de incorporación del enfoque de género en la docencia universitaria.

\section{Código 1.1 No se trabaja}

Los alumnos y las alumnas subrayan que la temática no ha sido abordada en sus clases.

\section{Código 1.2 Actividades puntuales}

Este código incluye las narrativas que evidencian que la igualdad de género se ha trabajado de manera puntual con algún tipo de actividad y al margen de lo programado en los temarios.

\section{Código 1.3 Integrado temario}

Se subraya que la temática objeto de estudio se ha trabajado de manera específica en el temario de una o varias asignaturas de la titulación que cursan.

Categoría 2. Opinión relevancia

Los códigos que integran esta categoría muestran la dualidad existente en el posicionamiento de los participantes, con respecto a la valoración que hacen del tratamiento del tema en las aulas universitarias.

\section{Código 2.1 Tema relevancia}

Este código aglutina una serie de subcódigos que justifican la defensa del alumnado del trabajo de esta temática en la educación superior. Entre sus argumentos figuran: 2.1.1 Formación integral, 2.1.2 Impulso cambio, 2.1.3 Lucha discriminación, 2.1.4 Mejora capacitación y 2.1.5 Tema actualidad.

\section{Código 2.2 Tema accesorio}

A diferencia del código anterior, en este caso se incluyen las aportaciones que demuestran que, desde el punto de vista de determinados estudiantes, no debería incluirse el enfoque de género en la docencia universitaria.

\section{Categoría 3. Dificultades}

La categoría engloba los fragmentos de texto que aluden a las dificultades y limitaciones que pueden localizarse en la incorporación de esta temática en las aulas de educación superior. 


\section{Código 3.1 Existencia dificultades}

Considera las aportaciones en las que los participantes identifican la existencia de una serie de dificultades. Entre ellas: 3.1.1 Rigidez temarios, 3.1.2. Tema controvertido, 3.1.3 Falta formación y sensibilización profesorado y 3.1.4 Escasez mujeres.

\section{Código 3.2 Inexistencia dificultades}

El código incluye los comentarios que obvian la existencia de dificultades y entienden que la incorporación del enfoque de género a la docencia universitaria es una cuestión necesaria.

\section{Categoría 4. Propuestas}

Esta categoría considera las propuestas que plantea el alumnado para trabajar la igualdad de oportunidades de género.

\section{Código 4.1 Actividades grupales}

Se sugiere la organización de actividades grupales en las que se tenga en cuenta el número de mujeres y hombres que componen los grupos para favorecer el trabajo cooperativo.

\section{Código 4.2 Reflexión y sensibilización}

Consideran apropiado diseñar situaciones para trabajar el tema de manera reflexiva y con la intención de favorecer la sensibilización del alumnado.

\section{Código 4.3 Incorporación temarios}

Se indica la conveniencia de incorporar el tema en los programas de una o varias asignaturas de las titulaciones que cursan.

\section{Código 4.4 Charlas y películas}

Se entiende apropiado promover la organización de actividades intercentros que contribuyan a la sensibilización con la temática.

\section{Código 4.5 Actitud profesorado}

Se propone incidir sobre la necesidad de un cambio de actitud en el profesorado, quien consideran debería dedicarle más tiempo y cuidar algunos aspectos como, por ejemplo, el uso de un lenguaje inclusivo.

\section{Código 4.6 Referentes}

El código incluye argumentos que defienden la conveniencia de mostrar testimonios que sirvan de referencia a los estudiantes para concienciarse sobre la importancia del tema. 